\title{
Tutorial: The Quantum Finite Square Well and the Lambert W Function
}

\begin{tabular}{|r|l|}
\hline Journal: & Canadian Journal of Physics \\
\hline Manuscript ID & cjp-2016-0602 \\
\hline Manuscript Type: & Tutorial \\
\hline Date Submitted by the Author: & 22-Aug-2016 \\
\hline Complete List of Authors: & $\begin{array}{l}\text { Roberts, Ken; University of Western Ontario, Department of Physics and } \\
\text { Astronomy } \\
\text { Valluri, Sree Ram; University of Western Ontario, Physics and Astronomy; } \\
\text { King's University College, Mathematics }\end{array}$ \\
\hline Keyword: & $\begin{array}{l}\text { Quantum Wells, Square Well Potential, Lambert W function, } \\
\text { Nanostructures, Conformal Mappings }\end{array}$ \\
\hline &
\end{tabular}

SCHOLARONE $^{\text {m }}$
Manuscripts 


\title{
Tutorial: The quantum finite square well and the Lambert $W$ function
}

\author{
Ken Roberts ${ }^{1}$ and S. R. Valluri ${ }^{2}$
}

\begin{abstract}
We present a solution of the quantum mechanics problem of the allowable energy levels of a bound particle in a one-dimensional finite square well. The method is a geometric-analytic technique utilizing the conformal mapping $w \rightarrow z=w e^{w}$ between two complex domains. The solution of the finite square well problem can be seen to be described by the images of simple geometric shapes, lines and circles, under this map and its inverse image. The technique can also be described using the Lambert $\mathrm{W}$ function. One can work in either of the complex domains, thereby obtaining additional insight into the finite square well problem and its bound energy states. This suggests interesting possibilities for the design of materials that are sensitive to minute changes in their environment such as nano structures and the quantum well infrared photodetector (QWIP).
\end{abstract}

\section{Introduction}

Quantum well models are important for the design of semiconductor devices, such as the quantum well infrared photodetector (QWIP) which is used for infrared imaging applications; see Schneider and Liu [1] for an overview. The QWIP relies upon a quantum well which has been sized so that the energy of an electron in the first excited state is quite near the threshold of confinement in the well. The QWIP is therefore very sensitive to the arrival of a single photon. There are many other uses of quantum well models in nanostructures; the textbook by Harrison [2] is a good survey of the field.

The one-dimensional quantum finite square well (FSW) model is a familiar topic in most introductory quantum mechanics books; see for instance Bransden and Joachain [3], section 4.6. After deriving a pair of equations to describe the bound energy levels within the well, the solution is carried out by graphical or computational methods. It is sometimes said that the FSW problem does not have an exact solution, but there are in fact exact solutions as presented in the papers of Burniston and Siewert [4-6] and others $[7-9,17-19]$. Those exact methods generally rely upon contour integration in the complex plane.

We have found a simple geometric method which can be used to describe the solutions of the onedimensional finite square well problem. Our approach is analytic, using complex variables, but does not rely

upon contour integration as such. We instead make a strong appeal to geometric imagination. We focus on the description of the solution set via conformal mapping of simple geometric shapes (lines and circles) between two complex domains, using the mapping given by $w \rightarrow z=w e^{w}$. Thus our solution might be described as "geometric-analytic". The method is presented in mathematical and physical detail to stimulate further research in this and related avenues.

Our paper is organized as follows. Section 2 provides the motivation for an analysis of this classic problem in quantum mechanics. In section 3 we show the mathematical details of our solution to the square well problem using the Lambert $\mathrm{W}$ function. The results of our analysis are discussed in section 4 and section 5 summarizes our conclusions

\section{Motivation}

When solving the one-dimensional FSW problem, after some initial definitions and discussion, a textbook will arrive at the task of finding solutions to one of the two equations

$$
v \tan v=u
$$

or

$$
v \cot v=-u
$$

\footnotetext{
${ }^{1}$ Physics and Astronomy Department, Western University, London, Canada, krobe8@uwo.ca

${ }^{2}$ Physics and Astronomy, and Applied Mathematics Departments, Western University, London, Canada; King's University College, London, Canada

An earlier version of this paper was published as ArXiv 1403.6685.
} 
together with the constraint

$$
u^{2}+v^{2}=R^{2} .
$$

Here $u$ and $v$ are positive reals which are related to the allowed bound energy levels which are to be found. $R$ is a unitless parameter of the problem which is determined only by the dimensions of the potential well - that is, its (spatial) width and (potential) depth - and independent of the energy level of the bound state. Bransden and Joachain call the parameter $R$ the "strength parameter" of the problem, and we will also use that terminology.

One is often shown graphical solutions of the pair of simultaneous equations (1) and (3), or of the pair (2) and (3), and is encouraged to use a computer to find numerical solutions. The allowable energy levels for the bound particle are then calculated from the values of $u$ or $v$.

In this note, we will show that the solutions of the FSW problem can be described using the Lambert W function [10-12]. This alternative solution may provide some insight into the FSW problem. Sometimes having a solution determined by an analytic function, instead of graphically or numerically, can make it easier to use that solution in subsequent work. For instance, it may be desirable to determine the sensitivity of the solution to changes in a parameter.

In contrast to the contour integral methods, our geometric-analytic method is quite simple to describe. The only concept which may be new will be the use of the Lambert W function, and we have tried to motivate that in context. The reader will find this solution of a practical problem, the quantum square well, provides a comprehensible introduction to the Lambert $\mathrm{W}$ function as one of the family of special functions which are useful not only for quantum problems but for a variety of problems in diverse fields.

\section{Solution using Lambert W}

This presentation of the solution of the finite square well (FSW) problem will write the solution in terms of complex variables as long as feasible, rather than introducing real and imaginary components too early.

Suppose a bound particle in a 1-dimensional finite square well potential $V(x)$, which is given by $V(x)=0$ for $x<-L$ or $L<x$, and by $V(x)=-V_{0}$ for $-L<x<L$. Here $L$ and $V_{0}$ are positive reals, $L$ being (half) the width of the well, and $V_{0}$ being the depth. The particle has energy $-E$, where $E$ is a positive real between 0 and $V_{0}$. The particle's wave function $\psi(x)$ satisfies the time-independent Schrödinger equation (TISE),

$$
\left(-\frac{\hbar^{2}}{2 m}\right) \psi^{\prime \prime}(x)+V(x) \psi(x)=-E \psi(x)
$$

Figure 1 shows the three regions: region 1 is $x<$ $-L$, with wave function $\psi_{1}$; region 2 is $-L<x<L$, with wave function $\psi_{2}$; region 3 is $L<x$, with wave function $\psi_{3}$.

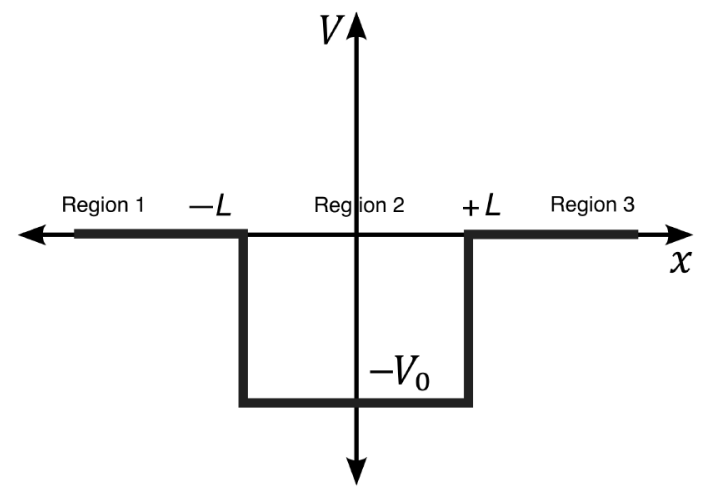

Figure 1: Square well.

In region 2, the TISE becomes

$$
\psi^{\prime \prime}(x)+\alpha^{2} \psi(x)=0
$$

where $\alpha^{2}=\left(2 m / \hbar^{2}\right)\left(V_{0}-E\right)$ and the wave function satisfies the equation

$$
\psi_{2}(x)=A e^{-i \alpha x}+B e^{i \alpha x}
$$

for some complex constants $A$ and $B$, to be determined. The value of $\alpha$ is real, and we can take it to be positive.

In regions 1 and 3 , the TISE becomes

$$
\psi^{\prime \prime}(x)-\beta^{2} \psi(x)=0
$$

where $\beta^{2}=\left(2 m / \hbar^{2}\right) E$. The value of $\beta$ can be taken to be positive real. The wave function satisfies the equation

$$
\psi_{1}(x)=D e^{\beta x}
$$

in region 1 , and the equation

$$
\psi_{3}(x)=C e^{-\beta x}
$$

in region 3 , for some complex constants $C$ and $D$, to be determined.

The continuity constraints can be expressed as four equations:

$$
\begin{aligned}
\psi_{2}(L) & =A e^{-i \alpha L}+B e^{i \alpha L} \\
& =C e^{-\beta L}=\psi_{3}(L)
\end{aligned}
$$




$$
\begin{gathered}
\psi_{2}(-L)=A e^{i \alpha L}+B e^{-i \alpha L} \\
=D e^{-\beta L}=\psi_{1}(-L) \\
\psi_{2}^{\prime}(L)=-i \alpha A e^{-i \alpha L}+i \alpha B e^{i \alpha L} \\
=-\beta C e^{-\beta L}=\psi_{3}^{\prime}(L) \\
\psi_{2}^{\prime}(-L)=-i \alpha A e^{i \alpha L}+i \alpha B e^{-i \alpha L} \\
=\beta D e^{-\beta L}=\psi_{1}^{\prime}(-L)
\end{gathered}
$$

We form linear combinations of these equations as follows: $i \alpha$ times equation (10) plus or minus equation (12), and $i \alpha$ times equation (11) plus or minus equation (13). Conceptually, those manipulations correspond to factoring

$$
-\left(\alpha^{2} \psi+\psi^{\prime 2}\right)=\left(i \alpha \psi+\psi^{\prime}\right)\left(i \alpha \psi-\psi^{\prime}\right)
$$

and requiring that one of the right-hand factors be zero, when evaluated at each of the points $x=-L$ and $x=L$. We obtain four new equations:

$$
\begin{aligned}
2 i \alpha B e^{i \alpha L} & =(i \alpha-\beta) C e^{-\beta L} \\
2 i \alpha A e^{-i \alpha L} & =(i \alpha+\beta) C e^{-\beta L} \\
2 i \alpha B e^{-i \alpha L} & =(i \alpha+\beta) D e^{-\beta L} \\
2 i \alpha A e^{i \alpha L} & =(i \alpha-\beta) D e^{-\beta L}
\end{aligned}
$$

One way to proceed is to divide equation (15) by equation (18), and realize that $B / A$ and $C / D$ are equal. Let $\epsilon$ denote $B / A$. Similarly, dividing equation (16) by equation (17) shows that $A / B$ also equals $C / D$. Hence $\epsilon$ equals $1 / \epsilon$, which leads to two cases: $\epsilon$ is either 1 or -1 .

$\epsilon$ equals $1 / \epsilon$. That corresponds to the conclusion that $\psi$ must be either an even function (also called an even parity solution) or an odd function (odd parity solution). If $\epsilon$ is 1 , then

$$
\psi_{2}(x)=A\left(e^{-i \alpha x}+e^{i \alpha x}\right),
$$

which is the even function $\cos (\alpha x)$ times a complex constant $2 A$, whose phase may be chosen arbitrarily. If $\epsilon$ is -1 , then

$$
\psi_{2}(x)=A\left(e^{-i \alpha x}-e^{i \alpha x}\right),
$$

which is the odd function $\sin (\alpha x)$ times a complex constant $-2 i A$, whose phase may be chosen arbitrarily.

The usual approach to find the solution of Bransden and Joachain [3], works with real values and hence trigonometric functions. That dichotomy eventually results in the two distinct equations (1) and (2). Here, we continue with a complex valued approach to the FSW problem to get the insights that brings. So we will simply for the time being let $\epsilon$ denote a value which, in the solution, will be either 1 or -1. That is, $\epsilon$ represents either of the two square roots of unity.

The four equations (15) through (18) reduce to two equations:

$$
\begin{aligned}
& \epsilon 2 i \alpha A e^{i \alpha L}=(i \alpha-\beta) C e^{-\beta L} \\
& 2 i \alpha A e^{-i \alpha L}=(i \alpha+\beta) C e^{-\beta L}
\end{aligned}
$$

Dividing equation (21) by (22) gives (since $\epsilon= \pm 1$, $-\epsilon= \pm 1)$

$$
\epsilon e^{2 i \alpha L}=\frac{\beta-i \alpha}{\beta+i \alpha}
$$

We introduce variables $u=\beta L$ and $v=\alpha L$ to express (23) as

$$
\epsilon e^{2 i v}=\frac{u-i v}{u+i v}=\frac{(u-i v)^{2}}{u^{2}+v^{2}}
$$

The values of $u$ and $v$ are related to the energy $E$ via $u^{2}=\left(2 m / \hbar^{2}\right) E L^{2}$, and $v^{2}=\left(2 m / \hbar^{2}\right)\left(V_{0}-E\right) L^{2}$, so if we know $u$ or $v$, then the energy $E$ can be determined. Moreover, $u^{2}+v^{2}=\left(2 m / \hbar^{2}\right) V_{0} L^{2}=R^{2}$, say. The values of $u$ and $v$ lie on an $R$-circle. Here $R$ does not depend upon the energy, but is a parameter of the FSW problem, depending only on the (spatial) width and (energy) depth of the potential well and called the "strength parameter" [3]. $R$ is unitless, and as will be seen, the number of solutions of the FSW problem will increase as the value of $R$ gets larger.

Equation (24) thus simplifies to

$$
(u-i v)^{2}=\epsilon R^{2} e^{2 i v}
$$

We introduce the Lambert W function. Some references to Lambert $\mathrm{W}$ properties and applications are [10-12]. This function is creating a renaissance in the solution of many problems in diverse fields. For the present purpose, it suffices to know that Lambert $W(z)$ is the analytic multi-branch solution of $W(z) e^{W(z)}=w e^{w}=z$, where $z$ is the complex argument of $W(z)$. That is, if we can manipulate an equation into the form $w e^{w}=z$, then the solution will be one or all of the branches of $w=W(z)$.

Comparing the Lambert function with the natural $\log$ arithm function $\log (z)$, we observe that they are closely related. $w=\log (z)$ is the multi-branch analytic function which solves the equation $e^{\log (z)}=$ 
$e^{w}=z$. The natural logarithm is very familiar, and it has many useful properties. Lambert $\mathrm{W}$ is similarly useful, once one learns to recognize problem situations where it has application.

Taking the square root of equation (25), one obtains

$$
u-i v= \pm \sqrt{\epsilon} R e^{i v}=\gamma R e^{i v}
$$

Here $\gamma$ represent the square root of $\epsilon$, as well as a \pm 1 factor which comes from taking the square root of $e^{2 i v}$. That is, there are four alternatives; $\gamma$ may be any of \pm 1 or $\pm i$. $\gamma$ is the fourth root of unity. Letting $\gamma$ represent an arbitrary member of that set of four alternatives is convenient, since for instance the conjugate, reciprocal or negative of the symbol $\gamma$ is just $\gamma$, that is, another representative of that set of four alternatives. From the conjugate of equation (26), one obtains a simpler-looking equation

$$
(u+i v) e^{i v}=\gamma R
$$

At this point replacing $e^{i v}$ by $\cos v+i \sin v$, would result in

$$
(u+i v)(\cos v+i \sin v)=\gamma R
$$

Supposing for instance that $\gamma=1$, and taking the imaginary part of equation (28) gives

$$
v \cos v+u \sin v=0
$$

which is

$$
v \cot v=-u
$$

which, together with the constraint that $u$ and $v$ lie on the circle of radius $R$, is the textbook version of the FSW solution. Keeping the solution process general for a bit longer reveals further insights.

Consider the geometric content of equation (27). Supposing $u$ and $v$ are positive, and writing $w=$ $u+i v$, the point $w$ lies in the first quadrant. Further, we know that the magnitude of $w$ is $R$, since $u^{2}+v^{2}=R^{2}$. The effect of the factor $e^{i v}$ in equation (27) is to rotate $w$ counterclockwise by an angle of at most $R$ radians, so that the product $w e^{i v}$ can lie in any quadrant. However, the condition that $w e^{i v}$ equal $\gamma$ (one of the four fourth roots of unity) times the real number $R$, means that only certain rotational angles $v$ are allowable as solutions of equation (27). The point $w e^{i v}$ cannot lie "within" a quadrant; it must lie on either the real or the imaginary axis. That of course is the resonance phenomenon which is familiar with regard to stabilizing the values of quantum mechanical observables.
The geometric description of equation (27) is connected to the Lambert $\mathrm{W}$ function. Consider the real and imaginary parts of the expression $z=w e^{w}$ where $w=u+i v$. We have

$$
\begin{gathered}
z=w e^{w}=(u+i v) e^{u+i v}=e^{u}(u+i v) e^{i v} \\
=e^{u}(u+i v)(\cos v+i \sin v) \\
=e^{u}(u \cos v-v \sin v)+i e^{u}(u \sin v+v \cos v)
\end{gathered}
$$

Imagine the mappings between the $z$-plane and the $w$-plane. The map $z=w e^{w}$ carries the $w$-plane to the $z$-plane, and the inverse map (multi-branch) is the Lambert $\mathrm{W}$ function, carrying the $z$-plane to the $w$-plane. The two rays from the origin in the $z$-plane along the imaginary axis, are the values of $\gamma r$ when $\gamma= \pm i$ and $r$ is a positive real. Those rays in the $z$-plane correspond to the $w$-plane values for which

$$
u \cos v-v \sin v=0
$$

which is equivalent to the equation

$$
v \tan v=u \text {. }
$$

Similarly, the two rays from the origin in the $z$ plane along the real axis, are the values of $\gamma r$ when $\gamma= \pm 1$ and $r$ is a positive real. Those rays in the $z$-plane corresponds to the $w$-values for which

$$
u \sin v+v \cos v=0
$$

which is equivalent to the equation

$$
v \cot v=-u \text {. }
$$

Finally, the $R$-circle in the $w$-plane, under the mapping $z=w e^{w}$, has its image as a closed (multiloop, self-intersecting) curve in the $z$-plane as shown in figure 3.

Those set correspondences show how to visualize the solution of the FSW problem in terms of the Lambert $\mathrm{W}$ function. There are two alternative approaches:

(A) Start with the axes (both real and imaginary) of the $z$-plane excluding the point at the origin. Let's call those two axes the sets $X$ and $Y$, and let their union be $S=X \cup Y$. That is, the set $S$ is four axial rays from the origin in the $z$-plane. Map the axial rays to the $w$-plane via the multi-branch Lambert $\mathrm{W}$ function, obtaining the set $W(S)=W(X) \cup W(Y)$ as a family of lines in the $w$-plane - one line for each combination of an axial ray and a branch of the Lambert $\mathrm{W}$ function. Intersect that set $W(S)$, in the $w$ plane, with the circle $|w|=R$. That is the solution 
set of the FSW problem.

Alternatively:

(B) Start with the circle $|w|=R$ in the $w$-plane, let's call it $P$. Map that circle to the $z$-plane via $z=w e^{w}$, to obtain a set, let's say $Q$, in the $z$-plane. In a set-wise notation, we might write $Q=P e^{P}$. Intersect that set with the axes $X$ and $Y$ in the $z$-plane. That also is the solution of the FSW problem, with the solution set represented in the $z$-plane.

It is useful to see these two approaches in graphical form.

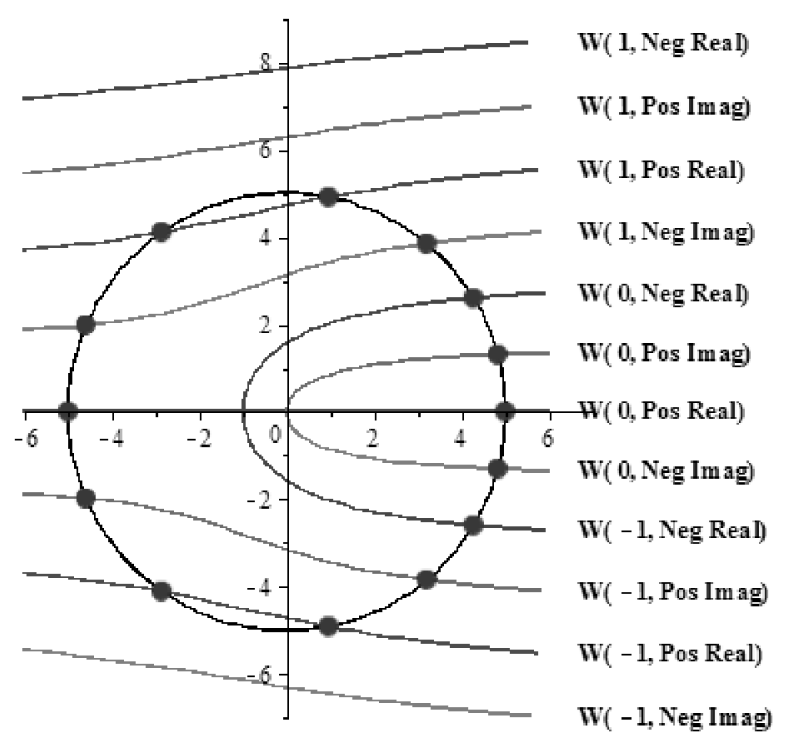

Figure 2: Solution in w-plane. $W(k$,ray $)$ means branch $k$ image of axial ray.

As an illustration, suppose that $R=5$. We will start with approach $\mathrm{A}$, and observe that it reproduces the textbook solution. Figure 2 shows the $w$-plane. The circle $|w|=5$ is shown, along with maps of the real and imaginary axes as transformed by the Lambert $\mathrm{W}$ function. Various branches of the Lambert W function have been shown, for branch numbers 1 to - 1 (using the conventional definitions of branch numbers from Corless, et al [10]). Only the branch numbers 1,0 and -1 intersect the circle $|w|=5$. The first quadrant of figure 2, for $u>0$ and $v>0$, is readily seen to be equivalent to the textbook diagram, with the $u$ and $v$ axes flipped. Compare for instance figure 4.12 of Bransden and Joachain [3]; the authors, in their figure, use symbols $\xi$ for $v, \eta$ for $u$, and $\gamma$ for $R$. Their $\gamma=5$ curve shows the four bound state solutions, two even states and two odd states, as visible in the first quadrant of our figure 2.
Now, what happens if instead we use approach B to look at the solution for the $R=5$ example, working in the $z$-plane? Instead of the circle $P:|w|=5$ in the $w$-plane we have its transform $Q$ under the map $z=w e^{w}$, which gives a closed curve in the $z$ plane, with multiple loops around the origin. We are interested in the places where that curve crosses one of the axes, since those are the possible solutions of the finite square well problem. Figures 3 to 6 show that curve, the whole of the $Q$ curve in figure 3, with magnifications in figures 4 to 6 to exhibit the fine details.

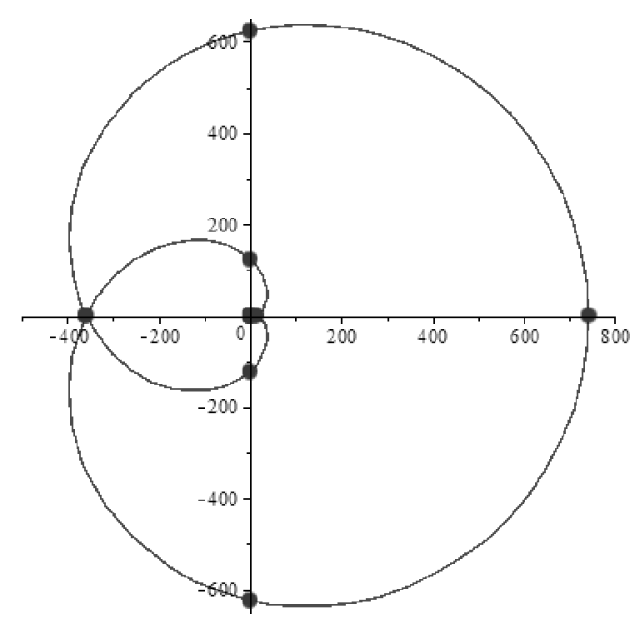

Figure 3: Solution in $z$-plane.

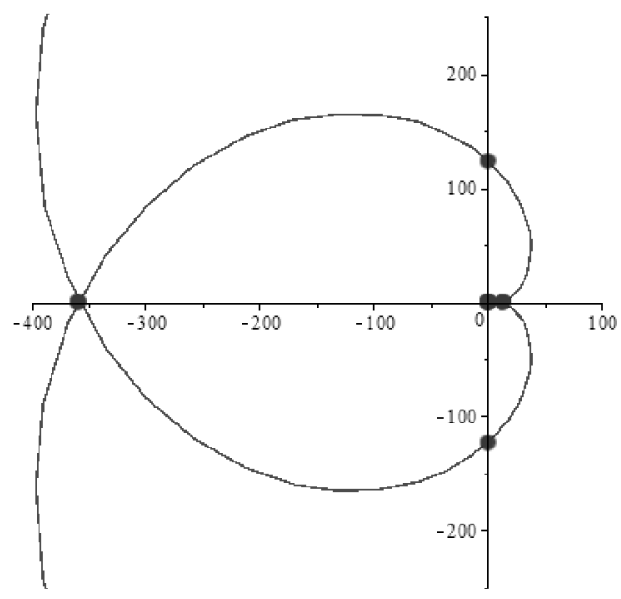

Figure 4: Solution in $z$-plane, magnified. 


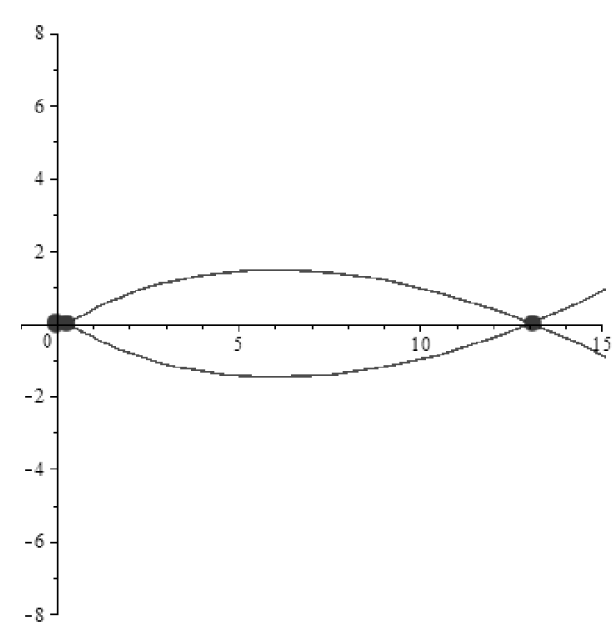

Figure 5: Solution in $z$-plane, magnified.

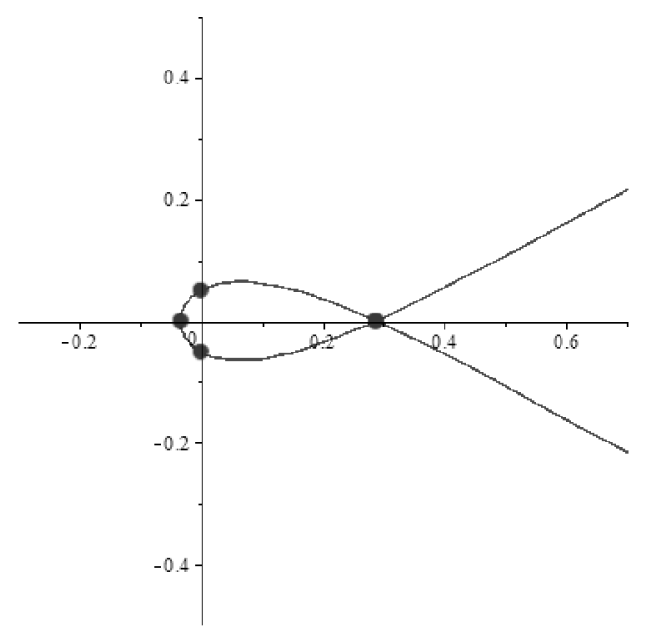

Figure 6: Solution in $z$-plane, magnified.

The $Q$ curve crosses the axes 14 times, counting multiplicities, ie crossings at the same coordinates but with different trajectories. Those correspond to the 14 solutions, the circle intersections in figure 2, of the problem visualized in the $w$-plane. To express those solutions in terms of the phase $\theta$ of the $w$ variable, write $w=R \exp (i \theta)$. Table 1 shows the solutions (the phase angle $\theta$ parameter values when the curve $Q$ crosses one of the axes).

\begin{tabular}{|c|c|}
\hline x-axis crossing & y-axis crossing \\
\hline 0.000 & 0.264 \\
0.546 & 0.875 \\
1.377 & 2.735 \\
2.179 & 3.548 \\
3.142 & 5.408 \\
4.105 & 6.019 \\
4.906 & \\
5.737 & \\
\hline
\end{tabular}

Table 1: Phase angle $\theta$ values at axis crossings.

To obtain the solutions in the first quadrant, that is with $u$ and $v$ required to be positive, we can simply restrict the $\theta$ phase to be between 0 and $\pi / 2$, and exclude the case $\theta=0$ as not being physical. That produces the standard textbook results.

As one can see the Lambert $\mathrm{W}$ function, considered as a mapping between two planes, provides a visualization of the solution of the finite square well problem in terms of simple geometric objects: the real and imaginary axes, and circles around the origin. The area of the circle around the origin corresponds to the dimensions of the finite square well, and is proportional to the square of the strength parameter, that is, to the depth of the well and to the square of its linear dimension. In symbols, $R^{2} \sim V_{0} L^{2}$. The proportionality constant is $2 m / \hbar^{2}$, and the units of that constant cancel the units of $V_{0} L^{2}$, resulting in $R^{2}$, and hence $R$, being unitless.

\section{Discussion}

The Lambert $\mathrm{W}$ description of the finite square well problem is best visualized as a conformal map between two complex planes, produced by the mapping $w \rightarrow z=w e^{w}$, the Lambert $\mathrm{W}$ function being the multi-branch inverse of that mapping.

The axial rays in the $z$-plane map to the Lambert $\mathrm{W}$ lines in the $w$-plane, whose intersections with the circle of radius $R$ about the origin give the solutions to the FSW problem. Alternatively, the circle of radius $R$ about the origin in the $w$-plane maps to a multi-loop closed curve in the $z$-plane, whose intersections with the axial rays also give the solutions to the FSW problem. Thus one may approach the problem situation working in either the $w$-plane (as is traditional), or in the $z$-plane - the choice of plane being determined by the convenience of other aspects 
of the particular problem which may be simpler in one or the other of those representations.

This technique bears some similarities to the method used in [12] to determine the fringing fields of a parallel plate capacitor, representative of problems that can be solved using the conformal group of transformations.

Because the mapping is conformal, the angles between the circle in figure 2 and the various Lambert $\mathrm{W}$ lines are equal to the angles of the corresponding intersections in figure 3 of the multi-loop image of the circle and the axial rays. That suggests some possibilities for design of materials to be sensitive to slight changes in their environment, and leads back to the topic of the quantum well infrared photodetector (QWIP) with which we introduced this paper.

It is worth noting that the finite square well is "realistic" despite its simplicity, and continues to find use in contemporary research. Harrison [2], chapter 2, describes applications of the finite square well in semiconductor device design. See also Miller [13], section 2.9. Deshmukh, et al [14] use a 3dimensional radial finite square well model to characterize the attosecond-scale time delays of the photoionization of an atom of Xenon trapped within a C60 fullerine molecule. Kocabas, et al [15], in a study of mathematical models for metal-insulatormetal waveguides, note (pages 13-14 of their paper) that there is a close relationship between the onedimensional Schrödinger equation and the electromagnetic wave equation in layered media, and mention several ideas for investigation. They write "It is intriguing to ask whether such studies [of FSW solutions and their relationship to changes in reflection spectra of wells] could be useful in optics for the investigation of the effects of material interfaces". Thus there is plenty of opportunity to do interesting work even with as old and familiar a topic as the finite square well.

There is a relationship of the solutions of the finite square well to the quadratix of Hippias, which is the solutions in the $(x, y)$ plane of the equation $x=y \cot y$. One can flip the axes or make translations to get other similar expressions of the relationship between the two variables. See Corless, et al [10].

The quadratix of Hippias can be used to solve various problems, such as the trisection of an arbitrary angle. Harper and Driskell [16] have an enjoyable description of construction of the quadratix, using interactive software for geometric constructions, and show how to use the quadratix to multiply an angle by any factor which can be expressed as a ratio of the lengths of two straight lines. That raises the entertaining possibility that one could perhaps present some aspects of quantum mechanics in the language of Euclidean geometry. Quantum mechanics is already often described with a visual, diagrammatic language.

We anticipate there may be other interesting aspects of this geometric-analytic solution technique.

\section{Conclusions}

We have presented a solution of the quantum mechanics problem of the allowable energy levels of a bound particle in a 1-dimensional finite square well potential. The solution is a "geometric-analytic" technique utilizing the Lambert $\mathrm{W}$ function. The solutions can be represented in either of two domains, and a representation in the transformed domain has a particularly simple geometry. More work on this vibrant field of the application of the Lambert $\mathrm{W}$ function is anticipated.

\section{Acknowledgements}

S.R. Valluri would like to thank King's University College for their generous support.

\section{References}

[1] H. Schneider and H. C. Liu. Quantum Well Infrared Photodetectors: Physics and Applications, Springer, 2007.

[2] P. Harrison. Quantum Wells, Wires and Dots: Theoretical and Computational Physics of Semiconductor Nanostructures, 3rd ed., Wiley, 2009.

[3] B. H. Bransden and C. J. Joachain. Introduction to Quantum Mechanics, 2nd ed., Prentice-Hall, 2000 .

[4] C. E. Burniston and C. E. Siewert. Proc. Cambridge Philos. Soc., 73, 111 (1973).

[5] C. E. Siewert and C. E. Burniston. J. Math. Anal. Appl., 43, 626 (1973).

[6] C. E. Siewert. J. Math. Phys., 19, 434 (1978).

[7] P. Paul and D. Nkemzi. J. Math. Phys., 41, 4551 (2000). 
[8] D. L. Aronstein and C. R. Stroud Jr. J. Math. Phys., 41, 8349 (2000).

[9] R. Blümel. J. Phys. A: Math. Gen., 38, L673 (2005).

[10] R. M. Corless, G. H. Gonnet, D. E. G. Hare. D. J. Jeffrey and D. E. Knuth. Adv. Comput. Math., 5, 329 (1996).

[11] F. W. J. Olver, R. F. Boisvert, D. W Lozier and C.W. Clark,eds. NIST Handbook of Mathematical Functions, Cambridge University Press, 2010. pp. 111.

[12] S. R. Valluri, D. J. Jeffrey and R. M. Corless. Can. J. Phys., 78, 823 (2000).

[13] D. A. B. Miller. "Quantum Mechanics for Scientists and Engineers", Cambridge University Press, 2008.
[14] P. C. Deshmukh, A. Mandal, A. S. Kheifets. V. K. Dolmatov and S. T. Manson, Phys. Rev. A., 89, 053424 (2014).

[15] S. E. Kocabas, G. Veronis. D. A. B. Miller and S. Fan, Phys. Rev. B., 79, 035120 (2009).

[16] S. Harper and S. Driskell. "An Investigation of Historical Geometric Constructions", Loci, 2010.

[17] N. I. Ioakimidis. J. Math. Anal. Appl., 129, 134 (1988).

[18] E. G. Anastasselou and N. I. Ioakimidis. J. Math. Phys., 25, 2422 (1984).

[19] E. G. Anastasselou and N. I. Ioakimidis. J. Math. Anal. Appl., 112, 104 (1985). 